\title{
Sensitivity Analysis and Parameter Tuning of a Sea-Ice Model
}

\section{Jong G. Kim \\ Paul D. Hovland}

Mathematics and Computer Science Division

Argonne National Laboratory

9700 S. Cass Avenue

Argonne, IL 60439-4844

\begin{abstract}
The values of many of the parameters in climate models are often not known with any great precision. We describe the use of automatic differentiation to examine the sensitivity of an uncoupled dynamicthermodynamic sea-ice model to various parameters. We also illustrate the effectiveness of using these sensitivity derivatives with an optimization algorithm to tune the parameters to maximize the agreement between simulated results and observational data.
\end{abstract}

\section{Introduction}

The sea-ice cover in polar regions plays an important role in modeling the Earth's climate system. For example, sea-ice acts as a powerful insulating boundary layer, reducing the atmosphere-ocean heat exchange and reflecting incoming solar energy on the surface of sea-ice covers. Also sea-ice is a major factor in the thermohaline circulations [MMM90],[Jac97]. Thus, seaice is capable of profoundly amplifying and modulating the climate variations in regional and global-scale climate systems. For this reason, sea-ice models are being presently coupled to global atmospheric and ocean general circulation models in Earth climate studies.

The accuracy of a sea-ice model depends upon the accuracy of several model parameters. Yet tuning parameter values of the climate system has been largely an ad hoc procedure, because of the high computational cost of evaluating multivariate parametric sensitivities. Early sensitivity studies of sea-ice models include the works of Maykut and Untersteiner [MU71] and Semtner [AJS76] with a one-dimensional thermodynamic sea-ice model. Full scale sensitivity studies of a dynamic-thermodynamic sea-ice model were carried out by Parkinson and Washington [PW79], Holland et al. [HMM93], and Chapman et al. [CWB ${ }^{+}$94]. In all of these studies, the in- 
vestigators calculate the sensitivities by the so-called finite difference (FD) method, which obtains the derivatives by dividing the response perturbation of the simulation to input parameter variation. Automatic differentiation (AD) provides an attractive alternative, since sensitivity derivatives can be computed with much greater accuracy and typically at a lower cost relative to finite difference approximations [Gri89, Gri00, BCK M96].

In this paper, we present $\mathrm{AD}$ as an alternative to the FD method for tuning a sea-ice model. Following a brief discussion of the model (Section 2) and the simulation conditions (Section 3), we discuss our experiments in evaluating the sensitivities of a dynamic-thermodynamic sea-ice model (Section 4). We also discuss the effectiveness of using AD-generated derivative codes coupled with minimization algorithms for tuning the model parameters to maximize the agreement between simulated result and observational data (Section 5). We conclude with a brief description of future work.

\section{Model Description}

Sea-ice models are comprised of two major components: thermodynamics and dynamics. Their formulations involve various diagnostic analyses of the interdependent physical and dynamical mechanisms to produce simulated seasonal changes of sea-ice. The thermodynamic routines of the code used in the study are essentially the same as those described by Hibler [WDH80], while the dynamic component is the elastic-viscous-plastic (EVP) model presented by Hunke and Dukowicz [HD97]. We summarize the main elements of the formulation here in order to underline the parameters used in the numerical experiments of sensitivity studies and the tuning process. The Fortran code of the sea-ice model used in the study was written in rectangular coordinates [ACM99]. Numerical experiments for Arctic sea-ice cover were performed with given atmospheric and oceanic forcing terms.

\subsection{Thermodynamic Component}

The thermodynamic model for the calculations of ice thickness change and open-water formation are discussed in detail by Maykut and Untersteiner [MU71], Semtner [AJS76], and Hibler [WDH80], computing the time-dependent ice thickness and vertical ice temperature profile in vertical grid intervals. The vertical ice changes are modified according to the energy transfers between the ice layer boundaries: the ocean mixed layer and the atmosphere-ice and snow-ice interfaces. A basic surface heat balance equation is given by

$$
\begin{aligned}
k \frac{\partial T}{\partial h}=(1-\alpha) F_{s \downarrow} & +F_{L \downarrow}+D_{1}\left|U_{G}\right|\left(T_{a}-T_{0}\right) \\
& +D_{2}\left|U_{G}\right|\left[q_{a}\left(T_{a}\right)-q_{s}\left(T_{0}\right)\right]-D_{3} T_{0}^{4},
\end{aligned}
$$


where $T_{0}$ is the surface temperature, $\alpha$ is the surface albedo, $T_{a}$ is the air temperature, $T_{w}$ is the water temperature, $U_{G}$ is the geostrophic wind, $q_{a}$ is the specific humidity of the air, $q_{s}$ is the specific humidity at the ice surface, $F_{s \downarrow}$ and $F_{L \downarrow}$ are the incoming shortwave and longwave radiation terms, $D_{1}$ and $D_{2}$ are the bulk sensible and latent heat transfer coefficients, $D_{3}$ is the Stefan-Boltzmann constant times the surface emissivity, $k$ is the ice conductivity, and $h$ is the ice thickness. Key parameters for the sensitivity studies are $D_{1}, D_{2}$, and $\alpha$.

\subsection{Dynamic Component}

Ice motion and deformation associated with vertical ice change modeled by the thermodynamic component are determined by the dynamics of a seaice model. Governing the dynamics component, the momentum balance equation is written for a mass of ice within a grid cell:

$$
m \frac{\partial \mathbf{U}}{\partial t}=-m f \mathbf{K} \times \mathbf{U}+\tau_{a}+\tau_{w}-m g \nabla H+\nabla \cdot \sigma,
$$

where $m$ is the ice mass per unit area, $\mathbf{U}$ is the ice velocity, $f$ is the Coriolis parameter, $\mathbf{K}$ is a unit vector normal to the surface, $H$ is the sea surface height, $g$ is the gravity acceleration, and $\sigma$ is the two-dimensional internal stress tensor. The forces due to air and water stresses, $\tau_{a}$ and $\tau_{w}$, are given by

$$
\begin{gathered}
\tau_{a}=\rho_{a} C_{a}\left|U_{G}\right|\left(U_{G} \cos \phi+\mathbf{K} \times U_{G} \sin \phi\right), \\
\tau_{w}=\rho_{w} C_{w}\left|U_{w}-\mathbf{U}\right|\left[\left(U_{w}-\mathbf{U}\right) \cos \theta+\mathbf{K} \times\left(U_{w}-\mathbf{U}\right) \sin \theta\right],
\end{gathered}
$$

where $U_{G}$ is the geostrophic wind, $U_{w}$ is the ocean current, $C_{a}$ and $C_{w}$ are the air and water drag coefficients, $\rho_{a}$ and $\rho_{w}$ are the air and water densities, and $\phi$ and $\theta$ are the turning angles at the ice-air and ice-ocean interfaces. The EVP rheology model that relates the internal ice stress $\sigma$ and the rates of strain $\epsilon$ is given by

$$
\frac{1}{E} \frac{\partial \sigma_{j i}}{\partial t_{e}}+\frac{1}{2 \eta} \sigma_{i j}+\frac{\eta-\zeta}{4 \eta \zeta} \sigma_{k k} \delta_{i j}+\frac{P}{4 \zeta} \delta_{i j}=\epsilon_{i j},
$$

where $P$ is the internal (or hydrostatic) ice pressure, $\zeta$ and $\eta$ are the bulk and shear viscosities, and $E$ is Young's modulus. The stress tensor $\sigma$ and the ice motion vector $\mathbf{U}$ are updated with the integration of Equation (1.2). In the integration, an effective model time scale of $\triangle t$ is discretized by the subcyclic time step of $\Delta t_{e}=\Delta t / N$. In our study, the number of subcyclic iterations is set to $N=72$. The pressure of the internal ice strength is determined by the ice thickness $h$ and the fraction of ice-covered area $A$, given by

$$
P=P^{*} h e^{[-C(1-A)]} \text {. }
$$


The ice strength in this equation depends on both the ice thickness $h$ and the ice area (or concentration) $A$. For this reason, a conservation law is derived for both quantities by applying a two-dimensional continuity equation. The continuity equations used in the study are the same as those described by Hibler [WDH80]. The major parameters for the sensitivity study are $C_{a}, C_{w}$, and $P^{*}$.

\section{Model Problem and Simulation Conditions}

The domain data and the external forcing variables were provided by Arbetter in a personal communication. The model included the area from the Greenland-Iceland-Norwegian sea to portions of the Bering sea, an Arctic domain discretized by the resolution of an $80 \mathrm{~km}$ Cartesian grid (see Figure 1). The forcing variables of $T_{a}, q_{s}, F_{s \downarrow}, F_{L \downarrow}$, and $U_{G}$ were derived from National Centers of Environmental Prediction reanalysis fields for 1992 at six hour intervals. A small nonzero constant was used for ocean currents. The domain boundaries were warped by Neumann boundary conditions. The constant ice thickness of $2 \mathrm{~m}$ and concentration of 0.5 were set for the initial conditions. The initial ice velocities were set to zero. For the details of the other input physical coefficients, see references [HMM93], [CWB $\left.{ }^{+} 94\right]$.

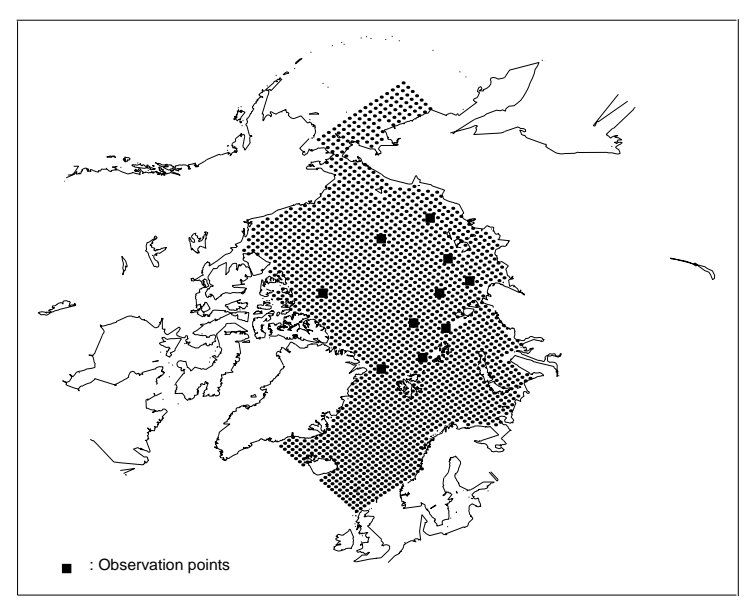

FIGURE 1. The model grid with resolution of $80 \mathrm{~km}$ and the locations of the observation data used in the study. 
TABLE 1.1. AD and FD derivatives of the average ice drift speed with respect to seven dynamic and thermodynamic parameters.

\begin{tabular}{|c|r|r|r|}
\hline Parameters & ADIFOR & FD, $\triangle=0.05$ & FD, $\triangle=0.001$ \\
\hline \hline$C_{a}$ & $0.36078 \mathrm{E}-01$ & $0.36247 \mathrm{E}-01$ & $0.36042 \mathrm{E}-01$ \\
$C_{w}$ & $-0.16748 \mathrm{E}-01$ & $-0.16185 \mathrm{E}-01$ & $-0.16736 \mathrm{E}-01$ \\
$P^{*}$ & $-0.16192 \mathrm{E}-01$ & $-0.15942 \mathrm{E}-01$ & $-0.16215 \mathrm{E}-01$ \\
$C$ & $-0.11839 \mathrm{E}-21$ & $0.69389 \mathrm{E}-15$ & $0.41633 \mathrm{E}-13$ \\
$D_{1}$ & $-0.17610 \mathrm{E}-02$ & $-0.16960 \mathrm{E}-02$ & $-0.17580 \mathrm{E}-02$ \\
$D_{2}$ & $0.54740 \mathrm{E}-04$ & $0.53690 \mathrm{E}-04$ & $0.53620 \mathrm{E}-04$ \\
$\alpha$ & $-0.58537 \mathrm{E}-11$ & $-0.58539 \mathrm{E}-11$ & $-0.58599 \mathrm{E}-11$ \\
\hline
\end{tabular}

\section{Sensitivity Experiments}

Sensitivity experiments were carried out for the dynamic-thermodynamic ice model parameters. As a dependent variable, the average ice velocity was calculated over ten simulation points, which were randomly distributed in the domain (shown in Figure 1). A two-month period of simulation (January and February 1992) was integrated for each sensitivity experiment. $\mathrm{AD}$ and FD derivatives for seven control parameters are summarized in Table 1.1. As the table shows, AD-computed derivatives for the average ice drift speed are comparable with FD-computed derivatives. However, with bigger increments of control parameters FD-derivatives are less accurate than AD-generated derivatives. Table 1.1 also indicates that the ice drift speed generally depends more on the dynamic parameters than on the thermodynamic parameters. The primary control parameters are the air and ocean drag coefficients determining the external stress terms in the dynamic equations. It is also indicated that the ice drift speed increases as the air drag coefficient increases. In contrast, the ice drift speed decreases with increasing ocean drag coefficient; this decrease occurs because the ocean currents impose a drag on the ice motion by reducing the motion force from wind stress. The effect of the ice strength constant is another substantial parameter for the sea-ice model. The ice motion tends to decrease with an increase of the ice strength constant. According to the ice rheology formulation, ice motion slows when the ice is compact with a bigger ice strength constant. The thermodynamic parameters provide weaker impact on the sea-ice model, perhaps because the formulations of ice dynamics are more complicated than those of thermodynamics. This result requires that more general sensitivity analysis be addressed with comprehensive thermodynamic formulations. 


\section{Parameter Tuning}

As demonstrated by the sensitivity results, the sea-ice model relies strongly on both dynamic and thermodynamic parameters. This interdependency of the model components requires accurately tuned parameters to simulate realistic average ice drift motions. Previous studies [HH97] have demonstrated the effectiveness of coupling optimization algorithms with automatic differentiation to tune algorithm parameters. This section describes the use of a similar approach to tune the physical parameters in the sea-ice model.

We used a bound constrained quasi-Newton minimization method, LBFGS-B [ZBLN94], which requires the user to provide the code computing the objective function (or cost function) and its gradients. The gradient information for the cost function was provided by the ADIFOR-generated derivative code. In this study, the cost function is defined by the norm of the difference between the observed and simulated average ice drift velocities. For the observational data, buoy ice drift velocities were provided by the International Arctic Buoy Programme (IABP) [CR93]. The five model parameters $C_{a}, C_{w}, P^{*}, \phi$ (or $\theta$ ), and $D 1$ were selected to control the simulated average ice velocities of ten separate points in the model domain (shown in Figure 1). The simulated average ice velocities were used to fit the observational data for a two-month period (January and February 1992). Five randomly selected parameter values $(4.3450 \mathrm{E}-04,0.0063$, $2640,0.2490,1.65^{\circ}$ ) were used for the initial guess of the minimization. The convergence behavior of the cost function is shown in Figure 2. The

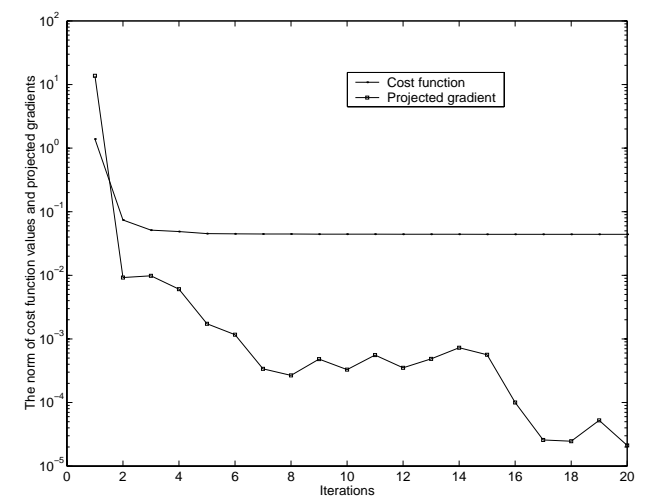

FIGURE 2. Convergence behavior of the cost function and its projected gradient with minimization iterations.

norm of the cost function was reduced by an order of magnitude in about 20 minimization iterations. The tuned parameter values $(0.0846,0.0031$, $50490,3.5927,53.1^{\circ}$ ) were obtained in the minimization process. The ratio between the two drag coefficients $C_{a} / C_{w}$, is 0.03654 . This is a relatively low 
value compared with another parameter set $(0.0055,0.0012,27500,2.284$, $25^{\circ}$ ), which has been used as default values in most sea-ice modeling studies. The ice motions simulated by these two different parameter sets are plotted in Figure 3. The ice motions of both are encouragingly comparable to the observed figures analyzed by IABP using buoy drift data (for the details of observed ice motions, see the web page of IABP [RO]). Figure 3 also indicates that the drift speed is overestimated by the default parameters. This overestimation is clearly observed in the region of Fram strait.
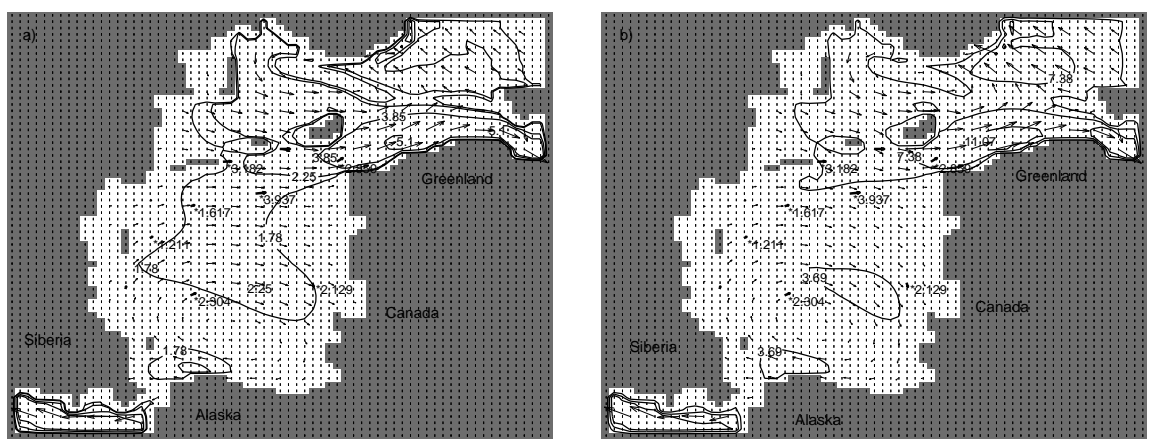

FIGURE 3. Average ice drift motion $(\mathrm{cm} / \mathrm{sec})$ plots for January and February 1992: (a) simulated with tuned parameters $\left(0.0846,0.0031,50490,3.5927,53.1^{\circ}\right)$ and (b) simulated with the default parameter set $(0.0055,0.0012,27500,2.284$, $\left.25^{\circ}\right) ; *$ marks and thick vector lines are observational data.

\section{Conclusion}

Our experiments indicate that automatic differentiation can provide accurate sensitivity analysis of a multivariate sea-ice model. Our results are similar to those from sensitivity experiments described by Holland et al. [HMM93] and Chapman et al. [CWB ${ }^{+}$94]. In general, we observed that the sea-ice model strongly depends on the dynamic parameters. In the parameter-tuning process with buoy drift data, we observed that the LBFGS-B minimization algorithm coupled with the AD derivative code yields parameter values that produce realistic results. Thus, an optimizationbased approach using the analytic derivatives computed by AD provides an effective basis for producing a model which better matches observational data. Future work includes tuning the parameters of multidecadal simulations of the sea-ice model, incorporating additional observational data, and applying the tuning process to other components of a GCM. 


\section{Acknowledgments}

This work was supported by the Mathematical, Information, and Computational Sciences Division subprogram of the Office of Advanced Scientific Computing Research, U.S. Department of Energy, under Contract W-31109-Eng-38.

We thank Todd Arbetter of the University of Colorado-Boulder for providing the Fortran program and the model problem sets. We are grateful to Michael Tobis for suggesting the idea of this sea-ice model parameter-tuning project. We are indebted to Ian Foster, Jay Larson, and John Taylor of Argonne National Laboratory for valuable discussions and assistance during the project. Finally, we thank Gail Pieper and Boyana Norris of Argonne National Laboratory for the careful review of the manuscript.

\section{References}

[ACM99] T. E. Arbetter, J. A. Curry, and J. A. Maslank. Effects of rheology and ice thickness distribution in a dynamic-thermodynamic sea-ice model. J. Phys. Oceanog., 29:2656-2670, 1999.

[AJS76] Jr. A. J. Semtner. A model for the thermodyanmic growth of sea-ice in numerical investigation of climate. J. Phys. Oceanogr., 6:379-389, 1976.

[BCKM96] Christian Bischof, Alan Carle, Peyvand Khademi, and Andrew Mauer. ADIFOR 2.0: Automatic differentiation of Fortran 77 programs. IEEE Computational Science $\&$ Engineering, $3(3): 18-32,1996$.

[CR93] R. L. Colony and I. Rigor. International Arctic buoy program data report for 1 January 199231 December 1992. Tech. Memo. APL-UW TM29-93, Appl. Phys. Lab., Univ. of Washington, Seattle, 1993.

$\left[\mathrm{CWB}^{+} 94\right]$ W. L. Chapman, W. J. Welch, K. P. Bowman, J. Sacks, and J. E. Walsh. Arctic sea-ice variability: Model sensitivities and a multidecadal simulation. J. Geophys. Res., 99:919-935, 1994.

[Gri89] Andreas Griewank. On automatic differentiation. In Mathematical Programming: Recent Developments and Applications, pages 83-108, Amsterdam, 1989. Kluwer Academic Publishers.

[Gri00] Andreas Griewank. Evaluating Derivatives: Principles and Techniques of Algorithmic Differentiation. SIAM, Philadelphia, 2000. 
1. Sensitivity Analysis and Parameter Tuning of a Sea-Ice Model

[HD97] E. C. Hunke and J. K. Dukowicz. An elastic-viscous-plastic model for sea-ice dynamics. J. Phys. Oceanog., 27:1849-1867, 1997.

[HH97] Paul D. Hovland and Michael T. Heath. Adaptive SOR: A case study in automatic differentiation of algorithm parameters. Technical Report ANL/MCS-P673-0797, Mathematics and Computer Science Division, Argonne National Laboratory, 1997.

[HMM93] D. M. Holland, L. A. Mysak, and D. K. Manak. Sensitivity study of a dynamic-thermodynamic sea-ice model. J. Geophys. Res., 98:2561-2586, 1993.

[Jac97] R. Jacob. Low frequency variability of the atmosphere-ocean system. PhD thesis, Univ. of Wisconsin, Madison, WI, 1997.

[MMM90] L. A. Mysak, D. K. Manak, and R. F. Marsden. Sea-ice anomalies observed in the Greenland and Labrador seas during 19011984 and their relationship to an interdecadal Arctic climate cycle. Climate Dyn., 5:111-133, 1990.

[MU71] G. A. Mykut and N. Untersteiner. Some results from a time dependent thermodynamic model of sea-ice. J. Geophys. Res., $76: 1550-1575,1971$.

[PW79] C. L. Parkinson and W. M. Washington. A large-scale numerical model of sea-ice. J. Geophys. Res., 84:311-337, 1979.

[RO] I. Rigor and M. Ortmeyer. Observations of ice SLP, SAT and ice motion. http://iabp.apl.washington.edu/Summary/.

[WDH80] III W. D. Hibler. Modeling a variable thickness sea-ice cover. Mon. Wea. Rev., 108:1943-1973, 1980.

[ZBLN94] C. Zhu, R. H. Byrd, P. Lu, and J. Nocedal. LBFGS-B: Fortran subroutines for large-scale bound constrained optimization. Report NAM-11, EECS Northwestern Univ., IL, 1994. 